\title{
SOME POPULATION GENETIC MODELS COMBINING SEXUAL SELECTION WITH ASSORTATIVE MATING
}

\author{
SAMUEL KARLIN* and PETER O'DONALD+ \\ * Department of Mathematics, Stanford University, Stanford, California 94305; \\ $\uparrow$ Department of Genetics, University of Cambridge, Downing Street, Cambridge CB2 3EH, \\ England
}

Received 15.ii.78

\begin{abstract}
Summary
Sexual selection can be defined in terms of mating preferences which favour particular genotypes or phenotypes and which are expressed at the same level in all females without any degree of assortment in the matings. In this paper, population genetic models are analysed in which assortative mating is combined with sexual selection. If separate mating preferences for each of three genotypes at a locus give rise to sexual selection and if each genotype may also mate assortatively, then a wide range of conditions entails the establishment of a unique, globally stable equilibrium which coincides with the equilibrium for assortative mating alone. Under certain other conditions, however, one of two different stable equilibria will be established dependent on the parameters of both sexual selection and assortative mating. This multiplicity of equilibria cannot arise in models involving either sexual selection or assortative mating alone. If females prefer either dominant or recessive phenotypes, then the advent of sexual selection prohibits the fixation that would occur by assortative mating alone: the effect of sexual selection is to ensure that a globally stable equilibrium will be established. In the combined model, therefore, sexual selection for a dominant trait exerts a greater effect on the final outcome than assortative mating. However, in contradistinction to the model with dominance, assortative mating usually determines the outcome when genotypes are preferred and assort separately.
\end{abstract}

\section{INTRODUGTION}

IN models of sexual selection (O'Donald, 1962, 1963, 1973a 1973b; for a general review see O'Donald, 1977a) some females are assumed to prefer to mate with particular males regardless of whether the females do or do not possess the preferred phenotype or genotype: the preference is expressed in all females equally; the phenotypes and genotypes do not assort together in the mating pairs of individuals. In models of assortative mating (O'Donald, 1960; Karlin, 1968; Karlin and Scudo, 1969; Scudo and Karlin, 1969) a certain proportion of the matings are assumed to take place between particular phenotypes or genotypes in excess of the matings that take place at random. This excess can be interpreted as the proportion of individuals expressing a mating preference for others like themselves. A mating preference is usually expressed only by females. If a preference for males with a particular phenotype or genotype is expressed only by females who possess the same phenotype or genotype, then assortative mating takes place.

In an organism that mates monogamously without any assortment of the mating preferences the overall frequencies of matings become the same 
as random mating frequencies, the genotypes following the HardyWeinberg Law. With monogamy, therefore, the existence of a mating preference can be detected only if some matings are assortative. Then mating frequencies will deviate from the expectations of random mating and genotypic frequencies will deviate from the Hardy-Weinberg Law. The existence of monogamous assortative mating usually implies some selection for change of gene frequency provided the mating preferences are determined by phenotype and some individuals are left unable to find mates, or some matings are less fertile than others (Karlin and Scudo, 1969; Scudo and Karlin, 1969). As Darwin (1871) suggested, matings later in the breeding season may be less successful in producing offspring: in many nonpasserine birds, breeding success declines as the breeding season advances. Preferred males are then selected because they find their mates sooner on average than the others. This can produce very strong selection (O'Donald, 1973a).

In polygynous mating systems, strong selection is the rule: the males, who have mated once, are available to mate again; the preferred males may therefore mate many times more often than the others. In extreme cases, a few dominant males sire a large proportion of the next generation. Selection for particular alleles will then be determined by preferences either randomly or assortatively distributed among the genotypes. We use the term sexual selection to describe mate selection by randomly distributed mating preferences and thus distinguish this process from that of assortative mating. Assortative mating and sexual selection always give rise to gene frequency selection in polygynous species. O'Donald (1977a) discussed these distinctions in more detail. Some qualitative and quantitative comparisons are set forth in Karlin and Farkash (unpub.).

Field observations (for example, Murton, Westwood and Thearle, 1973; Davis and O'Donald, 1976) can easily be made on assortative mating in monogamous species with a pair bond, since the numbers of different matings can be observed and counted directly. To observe sexual selection is much more difficult. Variation in the time spent finding a mate has been used to estimate sexual selection in the Arctic Skua, a monogamous sea bird (O'Donald, Wedd and Davis, 1974; O'Donald, 1977b; O'Donald and Davis, 1977). Direct observations in laboratory experiments have been used to determine the mating advantages of phenotypes and genotypes of Drosophila starting with the pioneering work of Sturtevant (1915) and leading up to Ehrman and Spiess' detailed and controlled experiments of frequencydependent mating choice for chromosomal inversions-for a review, see Ehrman (1972). In this paper we analyse models of polygynous mating systems in which sexual selection without assortment is combined with assortative mating. In a future paper we expect to analyse the corresponding models with monogamy.

These mixed models that we shall analyse may be applicable to data of the Arctic Skua. Davis and O'Donald (1976) analysed the numbers of matings of dark, intermediate and pale Arctic Skuas in the breeding populations of Fair Isle and Foula in Shetland. A model of assortative mating was fitted to the data with parameters $\alpha, \beta$ and $\gamma$ to measure the proportions of assortative matings of the three genotypes for darks, intermediates and pales. Fitting the parameter $\beta$ significantly reduces the heterogeneity in the Fair Isle data: $\alpha$ significantly reduces the heterogeneity 
in the Foula data. However, the heterogeneity in the assortative mating in the two populations is not significant. The parameter $\beta$ is sufficient to fit the data of both populations. At the same time, however, O'Donald, Wedd and Davis (1974) and O'Donald and Davis (1977) showed that both dark and intermediate male birds were favoured in sexual selection. Both assortative and non-assortative preferences produce selection of the allele for the melanic, intermediate and dark forms. A mixed model with monogamy and reduced fertility of the later matings describes the matings between the Arctic Skua genotypes.

Mixed models with polygyny may be appropriate for the description of mating preferences for particular Drosophila genotypes. Generally, the evidence from the experiments of Ehrman and Spiess (Ehrman, 1966, 1967, 1968, 1969, 1972; Spiess, 1968; Ehrman and Spiess, 1969; Spiess and Spiess, 1969) is consistent with models of sexual selection without assortment. O'Donald (1977c) fitted models of mating preferences to some of Ehrman and Spiess' data of mating success of males at different frequencies. There was no significant evidence for assortment in Spiess' data (Spiess, 1968; Spiess and Spiess, 1969) in which numbers of the females' genotypes were controlled in the mating choice experiments. However, specific mechanisms may give rise to assortment.

Drosophila may show assortment if genotypes are reared on different media: individuals tend to mate with individuals reared on the same media. This is an example of a widespread phenomenon in insects who return to specific food plants. In natural populations, therefore, assortative mating could easily be combined with sexual selection without assortment. This is the case we analyse in this paper.

\section{The MIXED ASSORTATIVE MATING-SEXUAL SELEGTION MODEL WITHOUT DOMINANCE}

\section{(i) Description of model}

The three genotypes determine separate phenotypes. As usual we assume a large population where mating preferences are exercised by the female, where males are polygamous ensuring that all females are fertilised, and where a $1: 1$ sex ratio prevails in reproduction. The assorting and sexual selection mating rates are described in terms of the following parameters.

\begin{tabular}{|c|c|c|c|c|}
\hline & $A A$ & $A a$ & $a$ & $\begin{array}{l}\text { Constraints on } \\
\text { the parameters }\end{array}$ \\
\hline \multirow[t]{2}{*}{ Sexual selection rates } & $\alpha$ & $\beta$ & $\gamma$ & $0 \leqq \theta+a \leqq 1$ \\
\hline & & & & $0 \leqq \theta+b \leqq 1$ \\
\hline Positive assortative rates & $a$ & $b$ & $c$ & $\begin{array}{c}0 \leqq \theta+c \leqq 1 \\
\text { where } \theta=\alpha+\beta+\gamma\end{array}$ \\
\hline
\end{tabular}

Let $u, v$, and $w$ be the frequencies of the genotypes $\mathrm{AA}, \mathrm{Aa}$ and aa respectively. The mating frequencies are listed in table $\mathrm{I}$. 
TABLE 1

Mating frequencies in the mixed model without dominance

\begin{tabular}{|c|c|c|c|}
\hline \multirow[b]{2}{*}{ Mating types } & \multicolumn{3}{|c|}{ Components of matings } \\
\hline & $\begin{array}{c}\text { Sexual } \\
\text { selection }\end{array}$ & $\begin{array}{c}\text { Positive } \\
\text { assortative } \\
\text { mating }\end{array}$ & $\begin{array}{l}\text { Random } \\
\text { mating }\end{array}$ \\
\hline $\begin{array}{l}A A \times A A \\
A A \times A a\end{array}$ & $\begin{array}{c}\alpha u \\
\alpha v+\beta u\end{array}$ & $\begin{array}{c}\text { au } \\
0\end{array}$ & $\begin{array}{c}(1-\theta-a) u^{2} \\
(2-2 \theta-a-b) u v\end{array}$ \\
\hline$A A \times a a$ & $\alpha w+\gamma u$ & 0 & $(2-2 \theta-a-c) u w$ \\
\hline$A a \times A a$ & $\beta v$ & $b v$ & $(1-\theta-b) v^{2}$ \\
\hline$A a \times a a$ & $\beta w+\gamma v$ & 0 & $(2-2 \theta-b-c) w w$ \\
\hline$a a \times a a$ & $\gamma w$ & $c w$ & $(1-\theta-c) w^{2}$ \\
\hline
\end{tabular}

The recurrence equations connecting genotype frequencies over two successive generations are then given by the expressions

$$
\begin{gathered}
u^{\prime}=\left(\alpha+\frac{\beta}{2}\right) p+a u+\frac{b}{4} v+(1-\alpha-\beta-\gamma) p^{2}-p\left(a u+\frac{b}{2} v\right) \\
v^{\prime}=\left(\gamma+\frac{\beta}{2}\right) p+\left(\alpha+\frac{\beta}{2}\right) q+2 p q(1-\alpha-\beta-\gamma)-p c w-q a u \\
w^{\prime}=\left(\gamma+\frac{\beta}{2}\right) q+c w+\frac{b}{4} v+q^{2}(1-\alpha-\beta-\gamma)-q\left(c w+\frac{b}{2} v\right) \\
p=u+\frac{v}{2}, \quad q=w+\frac{v}{2} .
\end{gathered}
$$

Henceforth, we restrict ourselves to the symmetric case

$$
\alpha=\gamma \quad a=c .
$$

This restriction must be unrealistic biologically, but we hasten to state that the qualitative results reflect the evolutionary consequences with general parameters (cf. Karlin, 1968 and Karlin and Farkash, unpublished).

Under the stipulation (2.2) the transformation equations can be represented more succinctly by the pair of equations

$$
\begin{aligned}
& u^{\prime}-w^{\prime}=(u-w)\left[1-\alpha-\frac{\beta}{2}+\frac{a-b}{2} v\right] \\
v^{\prime}= & \frac{1}{2}-\frac{(1-2 \alpha-\beta-a)}{2}(u-w)^{2}-\frac{a}{2}(1-v) .
\end{aligned}
$$

Case (1):

$$
\text { (ii) Results }
$$

$$
(a-b)(1-a)+(a-2)(2 \alpha+\beta)<0 .
$$

There is a unique globally stable equilibrium given by

$$
u^{*}=w^{*}=\frac{1}{2(2-a)}, \quad v^{*}=\frac{1-a}{2-a}
$$


extant independently of the sexual selection parameters. This is exactly the outcome from assortative mating and random mating alone when $b>a$.

With assortative mating alone the rate of approach to (2.3) occurs at a geometric rate governed by the multiplying factor

$$
\lambda=1+\frac{a-b}{2} \quad \frac{(1-a)}{2-a} .
$$

The superposition of sexual selection speeds the approach to the equilibrium (2.3) at the geometric rate

$$
\lambda=1+\frac{(a-b)}{2} \frac{(1-a)}{2-a}-\alpha-\frac{\beta}{2} .
$$

Moreover, the condition of Case (1) is certainly satisfied for $b>a$ and actually for a more extended range in the presence of partial sexual selection since the factor in the condition of Case (1), $(a-2)(2 \alpha+\beta)$, is negative.

It is perhaps surprising that in this case sexual selection has no effect on the degree of homozygosity, whereas it might be expected that the homozygosity would be reduced. Without assortment, sexual selection would give rise to the equilibrium frequencies $u^{*}=w^{*}=\frac{1}{4}, v^{*}=\frac{1}{2}$. Yet the equilibrium genotypic frequencies are exactly the same as when all preferential matings are assortative.

Case (2):

$$
(a-b)(1-a)+(a-2)(2 \alpha+\beta)>0
$$

Under this condition, fixation, which takes place when all preferential matings assort, is prohibited by the advent of sexual selection. The equilibrium of (2.3) exists but now is unstable.

There coexist two stable equilibria $\left(u_{1}^{*}, v_{1}^{*}, w_{1}^{*}\right)$ and $\left(u_{2}^{*}, v_{2}^{*}, w_{2}^{*}\right)$ explicitly given by (2.6) below.

Define

$$
\Delta=\frac{1-a+\frac{(a-2)(2 \alpha+\beta)}{a-b}}{1-2 \alpha-\beta-a}
$$

Then

$$
\begin{aligned}
& u_{1}^{*}=\frac{1-\hat{v}^{*}-\sqrt{\Delta}}{2}, \quad v_{1}^{*}=\frac{2 \alpha+\beta}{a-b}, \quad w_{1}^{*}=\frac{1-\hat{v}^{*}+\sqrt{\Delta}}{2} \hat{v}^{*}=\frac{2 \alpha+\beta}{a-b} . \\
& u_{2}^{*}=\frac{1-\hat{v}^{*}+\sqrt{\Delta}}{2}, \quad v_{2}^{*}=\frac{2 \alpha+\beta}{a-b}, \quad w_{2}^{*}=\frac{1-\hat{v}^{*}-\sqrt{\Delta}}{2}
\end{aligned}
$$

In Case (2) the relations at the start of the evolutionary process are either $w_{0}>u_{0}$ (or equivalently $p_{0}<\frac{1}{2}$ ) which defines the domain of convergence to

$$
\left(u_{1}^{*}, v_{1}^{*}, w_{1}^{*}\right)
$$

or $u_{0}>w_{0}\left(p_{0}>\frac{1}{2}\right)$ which defines the domain of convergence to

$$
\left(u_{2}^{*}, v_{2}^{*}, w_{2}^{*}\right)
$$


A numerical example giving the condition for Case (2) and the stable equilibria of (2.6) has values $a=c=\frac{1}{2}, b=0$ and $2 \alpha+\beta<\frac{1}{6}$. Table 2 gives the genotypic frequencies at equilibrium over a range of values of $2 \alpha+\beta$. For the values when $2 \alpha+\beta>\frac{1}{6}$ the condition for Case (1) holds

\section{TABLE 2}

Equilibrium genotypic frequencies in the mixed model without dominance

Homozygotes have equal assorting rates (given by $a=c=\frac{1}{2}$ ); heterozygotes do not assort $(b=0)$. Sexual selection is determined by mating preferences $\alpha=\gamma$ and $\beta$, giving a total proportion $2 \alpha+\beta$ of preferential matings.

\begin{tabular}{|c|c|c|c|c|}
\hline \multirow{2}{*}{$\begin{array}{l}\text { Preferential } \\
\text { matings } \\
2 \alpha+\beta\end{array}$} & \multicolumn{3}{|c|}{ Genotype frequencies } & \multirow{2}{*}{$\begin{array}{l}\text { Value of conditional } \\
\text { expression } \\
a(1-a)+(a-2)(2 \alpha+\beta)\end{array}$} \\
\hline & $u^{*}$ & $v^{*}$ & $w^{*}$ & \\
\hline 0.06 & 0.0136 & $0 \cdot 1200$ & $0 \cdot 8664$ & $0 \cdot 16$ \\
\hline $0 \cdot 08$ & $0 \cdot 0266$ & $0 \cdot 1600$ & $0 \cdot 8134$ & $0 \cdot 13$ \\
\hline $0 \cdot 10$ & $0 \cdot 0464$ & 0.2000 & 0.7536 & $0 \cdot 10$ \\
\hline $0 \cdot 12$ & 0.0765 & $0 \cdot 2400$ & 0.6835 & $0 \cdot 07$ \\
\hline $0 \cdot 14$ & $0 \cdot 1243$ & 0.2800 & 0.5957 & $0 \cdot 04$ \\
\hline $0 \cdot 16$ & $0 \cdot 2187$ & $0 \cdot 3200$ & 0.4613 & 0.01 \\
\hline $0 \cdot 18$ & $0 \cdot 3333$ & 0.3333 & $0 \cdot 3333$ & -0.02 \\
\hline $0 \cdot 20$ & $0 \cdot 3333$ & 0.3333 & $0 \cdot 3333$ & -0.05 \\
\hline
\end{tabular}

If the value of the conditional expression $a(1-a)+(a-2)(2 \alpha+\beta)<0$ then the single, globally stable equilibrium of Case (1) is reached. But if the condition for Case (2) holds with $a(1-a)+(a-2)(2 \alpha+\beta)>0$, then two different equilibria are stable: if $p_{0}<\frac{1}{2}$ then the equilibrium frequencies are given by the expressions for $u_{1}^{*}, v_{1}{ }^{*}$ and $w_{1}{ }^{*}$; if $p_{0}>\frac{1}{2}$ then the equilibrium frequencies are given by $u_{2}{ }^{*}, v_{2}{ }^{*}$ and $w_{2}^{*}$. In this table the equilibria of Case (2) are those for which $2 \alpha+\beta<0 \cdot 167$ : only the frequencies $u_{1}{ }^{*}, v_{1}{ }^{*}$ and $w_{1}^{*}$ are shown in the table; the frequencies $u_{2}{ }^{*}, v_{2}{ }^{*}$ and $w_{2}{ }^{*}$ can be found by interchanging the values of $u^{*}$ and $w^{*}$.

giving only one stable equilibrium at which $u^{*}=w^{*}$. The two stable equilibria of Case (2) do not arise in models of either assortative mating or sexual selection alone (cf. Karlin, 1968; O'Donald, 1977a). But if the phenotypes are determined not by each genotype separately but by levels of heterozygosity, then qualitative outcomes like those of (2.6) do occur (Karlin and Farkash, unpub.).

\section{(iii) Discussion}

(a) As mentioned earlier, the results with $a \neq b$ and/or $\alpha \neq \gamma$ involve more intricate formulae and conditions, but the qualitative possibilities completely parallel the descriptions of Cases (i) and (ii) under the symmetry postulate (2.2).

(b) In the combined model, assortative mating, rather than sexual selection, appears to be the factor that determines the equilibrium genotypic frequencies.

(c) Whereas with positive assortment alone, fixation may occur (e.g. when $a=c>b$ ), such monomorphisms are precluded by any degree of sexual selection except when certain rates of preference are zero (e.g. in some cases of $\alpha+\beta=0$ or if $\beta+\gamma=0$ ). 
(d) More than one stable polymorphic equilibrium may exist in the combined sexual selection and assortative mating model. This is not possible in the separate models. The dynamic evolution in this case depends crucially on initial conditions, founder effects and perhaps environmental correlates. Consistent with a multiplicity of stable equilibrium states is the interpretation that where genotype frequency varies over different localities a possible mechanism with respect to mating structure can involve the joint mechanisms of positive assortative mating and orders of sexual selection.

(e) In view of the interesting fact that for a wide spectrum of conditions, the unique globally stable equilibrium (2.3) for the combined model coincides with the equilibrium for the model of assortment alone; fitting equilibrium frequencies might therefore significantly underestimate the mating preferences. The difference can be partly sorted out by a series of longitudinal observations checking the rate of approach to equilibrium (compare (2.4) and (2.5) or the strength of the equilibrium over time under random perturbations).

\section{The MiXed ASSORTATIVE MATING-SEXUAL SELEGTION MODEL FOR A DOMINANT TRAIT}

\section{(i) Description of model}

There are two phenotypic classes conferred by a dominant and recessive trait, respectively. The assorting and sexual selection rates are as follows:

\begin{tabular}{lcccc} 
& \multicolumn{1}{c}{${ }^{\bar{A}}$} & & $\begin{array}{c}\text { Constraints on } \\
\text { the parameters }\end{array}$ \\
Sexual selection rates & $\rho$ & $\sigma$ & $0 \leqq \rho+\sigma+r \leqq 1$ \\
Positive assorting rates & $r$ & $s$ & $0 \leqq \rho+\sigma+s \leqq 1$
\end{tabular}

The assumptions on mating, reproduction and sex ratio are the same as in the previous section.

Let $u, v$ and $w$ be the frequencies of the genotypes $\mathrm{AA}, \mathrm{Aa}$ and aa, respectively. The mating frequencies are summarised in table 3.

TABLE 3

Mating frequencies in the mixed model for a dominant trait

\begin{tabular}{cccc} 
& \multicolumn{3}{c}{ Components of matings } \\
Mating types & Sexual selection & $\begin{array}{c}\text { Positive assortative } \\
\text { mating }\end{array}$ & Random mating \\
$A A \times A A$ & $\rho u^{2} /(1-w)$ & $r u^{2} /(1-w)$ & $(1-\rho-\sigma-r) u^{2}$ \\
$A A \times A a$ & $2 \rho u v /(1-w)$ & $2 r u v /(1-w)$ & $2(1-\rho-\sigma-r) u v$ \\
$A A \times a a$ & $\rho u w /(1-w)+\sigma u$ & 0 & $(2-2 \rho-2 \sigma-r-s) u w$ \\
$A a \times A a$ & $\rho v^{2} /(1-w)$ & $r v^{2} /(1-w)$ & $(1-\rho-\sigma-r) v^{2}$ \\
$A a \times a a$ & $\rho v w /(1-w)+\sigma v$ & 0 & $(2-2 \rho-2 \sigma-r-s) v w$ \\
$a a \times a a$ & $\sigma w$ & $s w$ & $(1-\rho-\sigma-s) w^{2}$
\end{tabular}




$$
\begin{aligned}
u^{\prime} & =\left[\frac{(\rho+r)}{1-w}+(1-\rho-\sigma-r)\right] p^{2} \\
v^{\prime} & =\frac{\rho\left(u q+\frac{v}{2}\right)}{1-w}+\frac{r v p}{1-w}+\sigma p+(1-\rho-\sigma) 2 p q-(r+s) p w-r v p \\
w^{\prime} & =(\rho+r) \frac{1}{4} \frac{v^{2}}{(1-w)}+\frac{\rho v w}{2(1-w)}+\sigma q+s w+(1-\rho-\sigma) q^{2}-\left(r \frac{v}{2}+s w\right) q
\end{aligned}
$$

and combining we have

$$
p^{\prime}=p\left[1-\frac{\rho+\sigma}{2}+\frac{\rho}{2(1-w)}+\frac{r-s}{2} w\right]
$$

\section{(ii) Results and interpretations}

(a) Whereas with partial assortment alone no stable polymorphism exists, the conjunction of sexual selection and assortative mating establishes a globally stable polymorphism. In this model sexual selection prohibits the fixation that always occurs by assortative mating alone.

In the case $\rho=0$, then either fixation of the genotype aa occurs, or a polymorphism is established at the frequency of a given by

$$
\tilde{w}^{*}=\frac{\sigma}{r-s}
$$

according to whether either $r<s+\sigma$ or $r>s+\sigma$ respectively holds. When $\sigma=0, \rho>0$; then genotype AA fixes if $r+\rho>s$ and otherwise a polymorphism results with

$$
\tilde{w}^{*}=\frac{s-(r+\rho)}{s-r} .
$$

(b) For $\rho \sigma>0$, the exclusive stable polymorphic equilibrium $(\tilde{u}, \tilde{v}, \tilde{w})$ has $\tilde{w}$ as the unique root in $(0,1)$ of the quadratic equation

$$
\sigma-(\rho+\sigma) w=(r-s) w(1-w) .
$$

(c) When $r=s$ (such that the dominant and recessive assorting rates are the same) the outcome coincides with that of sexual selection $\tilde{w}^{*}=\frac{\sigma}{\rho+\sigma}=\tilde{q}^{2}$ and $\tilde{u}=\tilde{p}^{2}, \tilde{v}=2 p \tilde{q}, \tilde{w}=\tilde{q}^{2}$.

(d) When $r=s=0$, the rate of approach to equilibrium is

$$
\lambda=1-\frac{(\rho+\sigma)^{2}}{\rho}\left(\sqrt{\frac{\sigma}{\rho+\sigma}}-\frac{\sigma}{\rho+\sigma}\right)
$$

and $0<\lambda<1$ (concerning details on (3.6), see Karlin, 1978).

An intricate analysis shows that the approach to the stable polymorphism is slower in the combined sexual selection-assortative mating model over that of (3.6) for sexual selection alone. 
(e) In contradistinction to the no-dominance model of Section 2, the sexual selection component for a dominant trait appears to exert greater influence on the evolutionary process in the combined model. Assortative mating alone for a dominant trait has no chance of producing a polymorphism, whereas the superposition of sexual selection will ensure a globally stable polymorphism.

It is of interest to compare the polymorphic equilibria for sexual selection without assortative mating

$$
w^{*}=\frac{\sigma}{\rho+\sigma}
$$

with the polymorphic equilibrium in the combined system in which the equilibrium frequencies are given by the solutions of the quadratic equation (3.5). The solutions are easily found as follows:

$$
\begin{aligned}
& \tilde{w}=\frac{\rho+\sigma+r-s-\sqrt{(\rho+\sigma+r-s)^{2}-4(r-s) \sigma}}{2(r-s)} \text { if } r>s \\
& \tilde{w}=\frac{\rho+\sigma+r-s+\sqrt{(\rho+\sigma+r-s)^{2}-4(r-s) \sigma}}{2(r-s)} \text { if } r<s .
\end{aligned}
$$

We have for $r>s, \tilde{w}<w^{*}$ and for $r<s$, then $\tilde{w}>w^{*}$. These are the relations we should expect: when $r>s$ and the dominant assorts more than the recessive, then the equilibrium frequency of the recessive is reduced by comparison with its frequency determined by sexual selection alone; when $r<s$, then its frequency is increased over that for sexual selection alone.

\section{REFERENGES}

DARWin, C. R. 1871. The Descent of Man, and Selection in Relation to Sex. John Murray, London.

DAVIS, J. W. F., AND O'DONALD, P. 1976. Estimation of assortative mating preferences in the Arctic Skua. Heredity, 36, 235-244.

ehrman, L. 1966. Mating Success and Genotype Frequency in Drosophila. Anim. Behav., 14, 334-339.

Ehrman, L. 1967. Further studies on genotype frequency and mating success in Drosophila. Amer. Natur., 101, 415-424.

EHRMAN, L. 1968. Frequency-dependence of mating success in Drosophila pseudoobscura. Genet. Res. Camb., 11, 135-140.

EHRMAN, L. 1969. The sensory basis of mate selection in Drosophila. Evolution, 23, 59-64. EHrman, L. 1972. Genetics and sexual selection. In Sexual Selection and the Descent of Man 1871-1971 (ed. B. Campbell), 105-135. Heinemann, London.

EHRMAN, L., AND SPIEss, E. B. 1969. Rare type mating advantage in Drosophila. Amer. Natur., 103, 675-680.

KARLIN, s. 1968. Equilibrium behaviour of population genetic models with nonrandom mating. 7. Applied Prob., 5, 231-313.

xARLIN, s. 1978. Comparisons of assortative mating and sexual selection models. I. Some one locus models for polygamous species. Theor. Pop. Biol. (in the press).

KARLIN, S., AND sCUDo, F. M. 1969. Assortative mating based on phenotype. II. Two autosomal alleles without dominance. Genetics, 63, 499-510.

MURTon, R. K., Westwood, N. J., AND THEARle, R. J. P. 1973. Polymorphism and the evolution of a continuous breeding season in the pigeon Columbia livia. 7. Reprod. Fert., Suppl., 19, 563-577.

o'DONALD, P. 1960. Assortative mating in a population in which two alleles are segregating. Heredity, 15, 389-396.

o'donald, P. 1962. Theory of sexual selection. Heredity, 17, 541-552. 
o'donald, P. 1963. Sexual selection for dominant and recessive genes. Heredity, 18, 451-457.

o'donald, P. 1973a. Frequency-dependent sexual selection as a result of variations in fitness at breeding time. Heredity, 30, 351-368.

o'Donald, P. 1973b. Models of sexual and natural selection in polygynous species. Heredity, $31,145-156$.

o'Donald, P. 1977a. Theoretical aspects of sexual selection. Theor. Pop. Biol., 12, 298-334. o'Donald, P. 1977b. Mating preferences and sexual selection in the Arctic Skua. II. Behavioral mechanisms of the mating preferences. Heredity, 39, 111-119.

o'Donald, P. 1977c. Mating advantage of rare males in models of sexual selection. Nature, Lond., 267, 151-154.

o'Donald, P., AND DAvIS, J. W. F. 1977. Mating preferences and sexual selection in the Arctic Skua. III. Estimation of parameters and tests of heterogeneity. Heredity, 39, 121-132.

O'DONALD, P., WEDD, N. S., AND DAVIS, J. W. F. 1974. Mating preferences and sexual selection in the Arctic Skua. Heredity, 33, 1-16.

scudo, F. M., AND KarLin, s. 1969. Assortative mating based on phenotype. I. Two alleles with dominance. Genetics, 63, 479-498.

SPIEss, E. B. 1968. Low frequency advantage in mating of Drosophila pseudoobscura karyotypes. Amer. Natur., 102, 363-379.

SPIESS, L. D., AND SPIESS, E. B. 1969. Minority advantage in inter-population matings of Drosophila persimilis. Amer. Natur., 103, 155-172.

STURTEVANT, A. 1915. Experiments on sex recognition and the problem of sexual selection. Anim. Behav., 5, 351-366. 